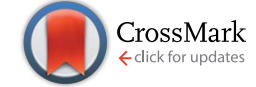

Cite this: RSC Adv., 2017, 7, 8415

Received 19th October 2016 Accepted 17th January 2017

DOI: 10.1039/c6ra25455a

www.rsc.org/advances

\section{Thermomechanical analysis of picograms of polymers using a suspended microchannel cantilever $\dagger$}

\author{
Syed Asad Manzoor Bukhari, M. Faheem Khan, Ankur Goswami, ${ }^{\star}$ Ryan McGee \\ and Thomas Thundat*
}

\begin{abstract}
Microchannel cantilevers are an emerging platform for physical characterization of materials at the picogram level. Here we report on detecting multiple thermal transitions in picogram amounts of two well-known polymers, semicrystalline poly(L-lactide) (PLA) and amorphous poly(methylmethacrylate) (PMMA), using this platform. The polymer samples, when loaded inside the cantilever, affect its resonance frequency due to changes in its total mass and stiffness. When taken through a thermal cycle, the resonance response of the cantilever further changes due to multiple thermal transitions of the samples. Continuous monitoring of the resonance frequency provides information about $\beta$-transition $\left(T_{\beta}\right)$, glass transition $\left(T_{g}\right)$, crystallization $\left(T_{c}\right)$, and melting $\left(T_{m}\right)$ of the confined polymer samples. The measured $T_{\mathrm{g}}, T_{\mathrm{c}}$, and $T_{\mathrm{m}}$ for PLA were $\sim 60,78$, and $154{ }^{\circ} \mathrm{C}$, respectively, while the $T_{\mathrm{g}}$ and $T_{\beta}$ for PMMA were 48 and $100{ }^{\circ} \mathrm{C}$, respectively. These results are in an agreement with the data obtained from differential scanning calorimetry (DSC). Because of its high sensitivity, this technique is capable of detecting the weaker $\beta$-transitions that cannot be observed with conventional DSC.
\end{abstract}

\section{Introduction}

Polymers are versatile materials with well-established applications in the pharmaceutical, ${ }^{\mathbf{1}}$ manufacturing, ${ }^{2}$ chemical, ${ }^{3}$ microelectronics ${ }^{4}$ and food industries. ${ }^{5}$ Recently, various researchers have reported the use of nanoscale amounts of polymers for applications such as non-volatile memory, ${ }^{6}$ energy storage, ${ }^{7}$ and biomimetics. ${ }^{8}$ Such applications require highly precise measurements of stability, durability, and purity of a polymer. Most of these characteristics can be investigated using thermal properties of polymers such as glass transition $\left(T_{\mathrm{g}}\right)$, crystallization $\left(T_{\mathrm{c}}\right)$, enthalpy relaxation, and melting $\left(T_{\mathrm{m}}\right){ }^{6-9}$ In addition to these well-known transitions, certain polymers may also exhibit a $\beta$-relaxation/transition, which is due to localized movements in the main chain or the movement of a very large side chain. ${ }^{\mathbf{1 0}}$ For large scale applications, most of these transitions can be probed with conventional techniques such as differential scanning calorimetry (DSC), ${ }^{11-13}$ dynamic mechanical analysis (DMA), ${ }^{\mathbf{1 4}}$ thermomechanical analysis (dilatometry), ${ }^{15}$ and dielectric spectroscopy (DES).$^{16}$ Although well established, these techniques are unsuitable for characterization of sub microgram levels of polymers due to the restrictions on the sample size $(\sim 5-10 \mathrm{mg})$ and dimensions.

Department of Chemical and Materials Engineering, University of Alberta, T6G 2R3, Edmonton, Canada.E-mail: agoswami@ualberta.ca; thundat@ualberta.ca

$\dagger$ Electronic supplementary information (ESI) available. See DOI: $10.1039 / \mathrm{c} 6 \mathrm{ra} 25455 \mathrm{a}$
Therefore, there is an emerging need to develop techniques capable of characterizing materials at the nanoscale. In order to fulfill this requirement, various sensors have been proposed to measure the thermal and mechanical properties of polymers at sub microgram levels. Microscale resonators is one such platform, which offers advantages such as; no shape dependence, high sensitivity, very small sample mass (nano to microgram), wide dynamic range of measurement, and faster operation. ${ }^{17-20}$ Micro resonators, such as microstrings and microcantilevers, have been previously employed for thermal analysis (of small masses) of polymers. ${ }^{17,18,21,22}$ Both of these techniques require special spray coating or ink jet printing to deposit nanograms of sample. ${ }^{23}$ Another challenge (when using microstrings or microcantilevers) is sublimation of the sample during heating. In order to overcome these difficulties, we have developed a microchannel cantilever consisting of microfluidic channel integrated on top of the cantilever. With the volume of the microchannel $\sim 50 \mathrm{pL}$, the resonance frequency of the cantilever is $\sim 150 \mathrm{kHz}$ while its quality factor lies in the order of six to eight thousand. These values ensure high resolution in detecting any changes in the resonance frequency. Previously, this principle and the device have been successfully employed to detect sub attogram mass, and the density and viscosity of picoliters of confined liquids and thermal sensor for binary liquid mixture. ${ }^{24-27}$ Without any complicated protocols or equipment, the polymer samples can be loaded into the microchannel using melt infiltration. In order to detect thermal transitions, the polymer-filled cantilever is heated under high 
vacuum. Although the cantilever is operated at low pressure $(\sim 5$ $\times 10^{-6} \mathrm{mbar}$ ), the polymer is enclosed within the microchannel which keeps the sample at atmospheric pressure. The incorporation of this microchannel enables the measurement of all thermal transitions up to (and including) the melting point. As a result, multiple heating cycles can be used to probe the transitions of the material in order to study its reversibility/ hysteresis. To test the cantilever systematically (as a platform for thermal analysis), two different polymers were chosen as a case study such as biodegradable, semi-crystalline poly (L-lactide) (PLA), and amorphous poly(methylmethacrylate) (PMMA). The aim of this study was to use a miniature, robust, and sensitive platform to obtain accurate information about the thermal events occurring in the polymers. Thermal characteristics such as $T_{\mathrm{g}}, T_{\mathrm{c}}, T_{\mathrm{m}}$ and $T_{\beta}$ are investigated and the results are compared to those obtained from conventional DSC.

\section{Experimental section}

Poly(L-lactide) or PLA $\left(M_{\mathrm{n}}=50000\right)$ and poly (methylmethacrylate) or PMMA $\left(M_{\mathrm{w}}=120000\right)$ were used as test materials due to their well-known thermal transitions. The densities of the PLA and the PMMA samples used in all experiments are $1.3 \mathrm{~g} \mathrm{~cm}^{-3}$ and $1.2 \mathrm{~g} \mathrm{~cm}^{-3}$ respectively. ${ }^{28,29}$

The microchannel cantilevers were fabricated using conventional top-down microfabrication techniques, as described in our previous study. ${ }^{21}$ The dimensions of the cantilevers were $200 \mu \mathrm{m}$ (length) $\times 20 \mu \mathrm{m}$ (width) $\times 3 \mu \mathrm{m}$ (microchannel height) as shown in Fig. 1a. The calculated mass of the cantilevers were 14.6 pg (considering $\rho_{\mathrm{Si}_{3} \mathrm{~N}_{4}}=3.44 \mathrm{~g} \mathrm{~cm}^{-3}$ ). Prior to loading the

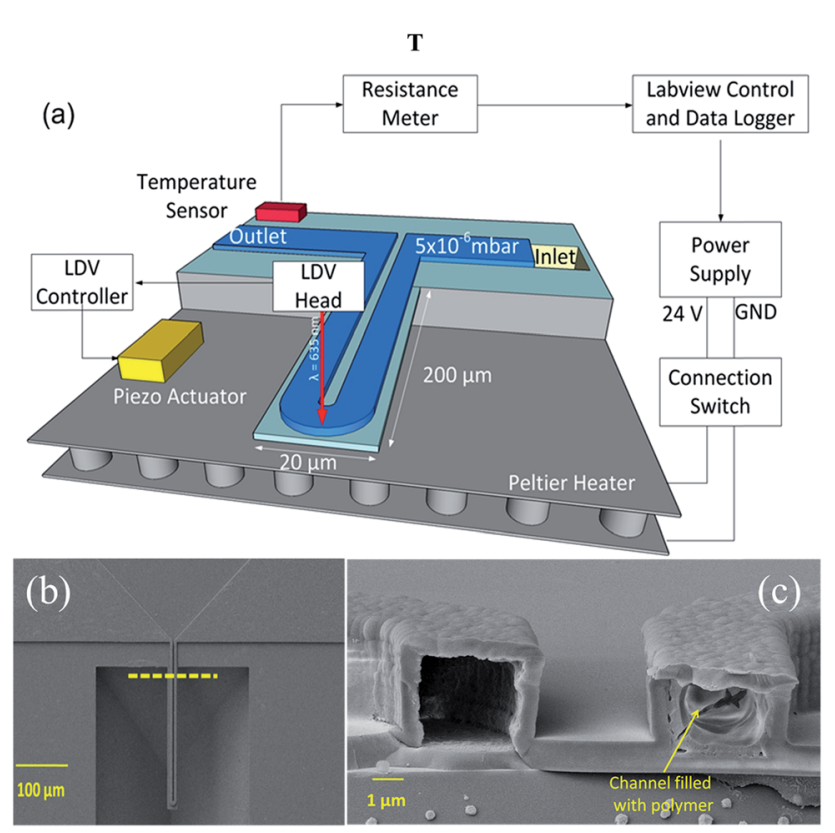

Fig. 1 (a) Experimental setup to characterize picograms of polymers. (b) SEM of the top view of the microchannel cantilever. Yellow dash line in shows from where the cantilever was cut to show (c). (c) Shows SEM of the cross-section of half-filled cantilever which depicts the unfilled and filled channel. polymer samples, each cantilever was cleaned with piranha (in order to remove organic contaminants) and heated on a hot plate at $200{ }^{\circ} \mathrm{C}$ for two hours to drive out any residual moisture. The cantilevers were filled with the polymer samples through a melt infiltration technique, where a small amount of the samples was placed on inlets (located on backside on the chip). The chips were heated on a hot plate to $150-170{ }^{\circ} \mathrm{C}$. At the melting temperature, due to strong capillary forces, the polymer is able to flow inside the microchannel.

Fig. 1a shows a schematic of the microchannel cantilever based thermal analysis platform used in this study. In order to apply a controlled thermal cycle, the cantilever was firmly placed on top of a Peltier element, and the temperature was continuously monitored by a Pt-1000 resistance temperature detector (RTD) mounted on top of the chip. Temperature was logged in real time by monitoring the RTD's resistance using a Keithley 197 multimeter interfaced with LabView software. The cantilever was heated from room temperature to $180^{\circ} \mathrm{C}$ at a heating rate of $4{ }^{\circ} \mathrm{C} \mathrm{min}^{-1}$. In order to enhance the signal to noise ratio (SNR), the cantilever was mechanically excited by an external piezo actuator. The resonance frequency was measured optically using a laser Doppler vibrometer (LDV, Polytec, USA) and was monitored continuously during the experiments. As the polymer samples are viscous, a cantilever cannot be effectively flushed and be used for another sample. Therefore, to avoid cross contamination, two similar cantilevers were used to characterize the two polymer samples. Prior to loading each sample, the empty cantilevers were characterized to determine their resonance frequency and phase. In an effort to enhance the quality factor (and thus the sensitivity) of the cantilevers, all the experiments were carried out at a vacuum level of $\sim 10^{-6}$ mbar. SEM of the top view of the cantilever is shown in Fig. 1b. A cross sectional SEM view of the channel (showing a filled and an empty channel) is shown in Fig. 1c. For reference measurements, the polymers were characterized using DSC (TAQ100) with 5-7 $\mathrm{mg}$ of sample, with a heating rate of $10^{\circ} \mathrm{C} \mathrm{min}^{-1}$.

\section{Results and discussion}

Though the cantilever has a microchannel fabricated on the top, it follows the same dynamics of vibration as a plain cantilever. The fundamental resonance frequency, $f_{\mathrm{c}}\left(T_{0}\right)$, of the cantilever at temperature, $T_{0}$, is given by eqn (1), where $k_{\mathrm{c}}$ and $m_{\mathrm{c}}$ are the cantilever's stiffness and mass respectively. ${ }^{30}$

$$
f_{\mathrm{c}}\left(T_{0}\right)=\frac{1}{2 \pi} \sqrt{\frac{k_{\mathrm{c}}}{m_{\mathrm{c}}}}
$$

Filling the cantilever with a polymer changes the stiffness and the mass of the cantilever, causing a shift in its resonance frequency. If the polymer stiffness and mass are indicated as $k_{\mathrm{p}}$ and $m_{\mathrm{p}}$, respectively then by assuming a parallel spring-mass model, the effective resonance frequency $f_{\mathrm{c}+\mathrm{p}}\left(T_{0}\right)$ of the polymer-filled cantilever would follow eqn (2). 


$$
f_{\mathrm{c}+\mathrm{p}}\left(T_{0}\right)=\frac{1}{2 \pi} \sqrt{\frac{k_{\mathrm{c}}+k_{\mathrm{p}}}{m_{\mathrm{c}}+m_{\mathrm{p}}}}
$$

In our case, since $\frac{k_{\mathrm{c}}}{m_{\mathrm{c}}}>\frac{k_{\mathrm{c}}+k_{\mathrm{p}}}{m_{\mathrm{c}}+m_{\mathrm{p}}}$, it is evident from eqn (1) and (2) that $f_{\mathrm{c}}\left(T_{0}\right)>f_{\mathrm{c}+\mathrm{p}}\left(T_{0}\right)(\mathrm{ESI} \dagger)$.

As compared to the structural material (silicon nitride) of the cantilever, the polymer exhibits a higher change in its stiffness due to heating. Therefore, as the temperature increases, the change in $k_{\mathrm{p}}$ dominates over $k_{\mathrm{c}}$, due to significant changes in the elastic modulus of the polymer. Hence, by monitoring $f_{\mathrm{c}+\mathrm{p}}(T)$, the transitions of the polymer can be determined. From eqn (3) it is evident that $k_{\mathrm{p}}$ can be determined by monitoring the resonance frequency of a cantilever before and after loading the polymer (ESI for detailed derivation $\dagger$ ).

$$
k_{\mathrm{p}}(T)=k_{\mathrm{c}}\left[\left(\frac{f_{\mathrm{c}+\mathrm{p}}(T)}{\left.f_{\mathrm{c}}(T)\right|_{T=T_{0}}}\right)^{2} C^{2}-1\right]
$$

here, $C=\left(1+\frac{m_{\mathrm{p}}}{m_{\mathrm{c}}}\right)^{-\frac{1}{2}}$. In this case $m_{\mathrm{c}}, m_{\mathrm{PLA}}$, and $m_{\mathrm{PMMA}}$ are calculated as 14.6, 6.24, and $2.8 \mathrm{pg}$ respectively. Since the samples are confined inside the microchannel, there is no mass loss due to sublimation.

Cantilevers with similar mechanical properties were used in order to perform the thermal analyses of the polymers. Prior to loading the polymers, resonance frequency and phase response of the empty cantilevers were recorded (shown in Fig. 2a and b).
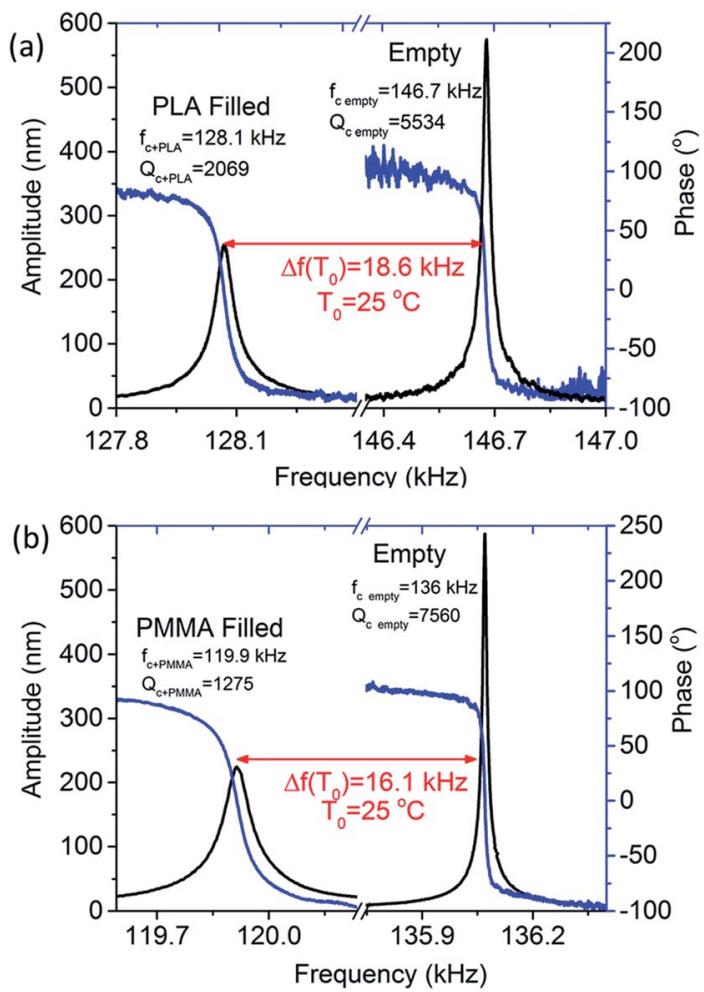

Fig. 2 Resonance frequency and phase of a microchannel cantilever filled with (a) PLA (b) PMMA.
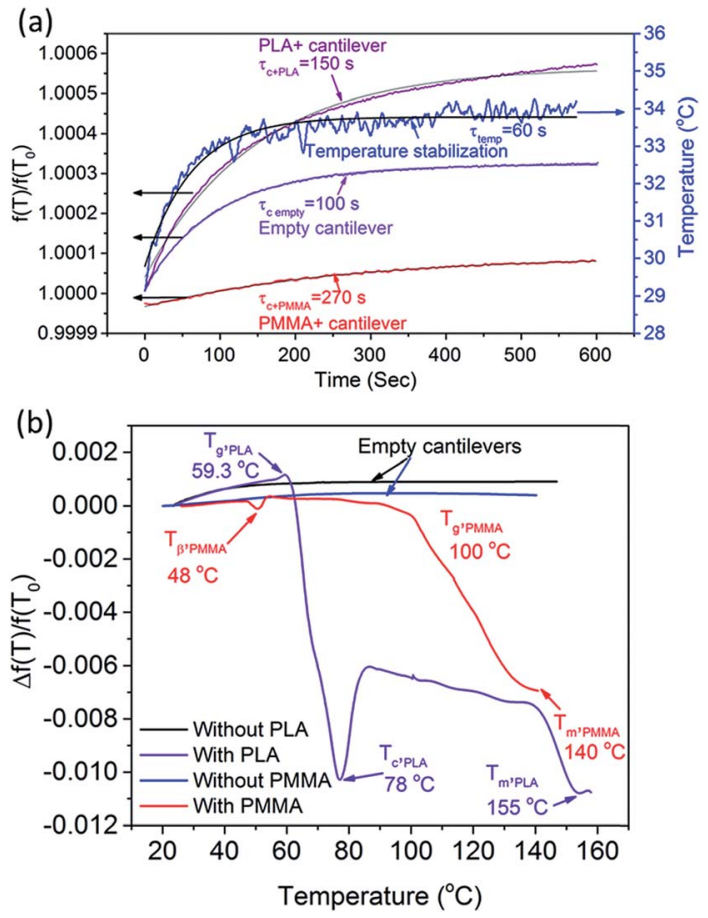

Fig. 3 (a) Stabilization in temperature and frequency measured for empty, PLA-filled, and PMMA-filled cantilevers. (b) Thermal characterization of empty, PLA-filled, and PMMA-filled cantilevers.

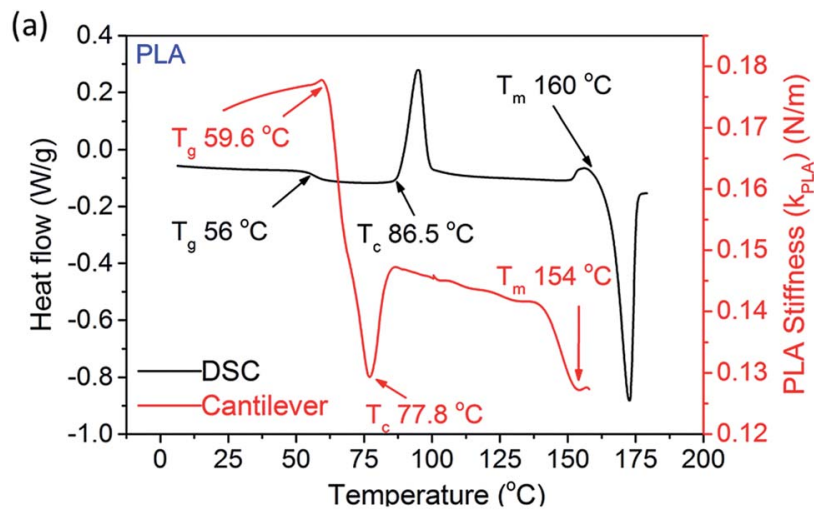

(b)

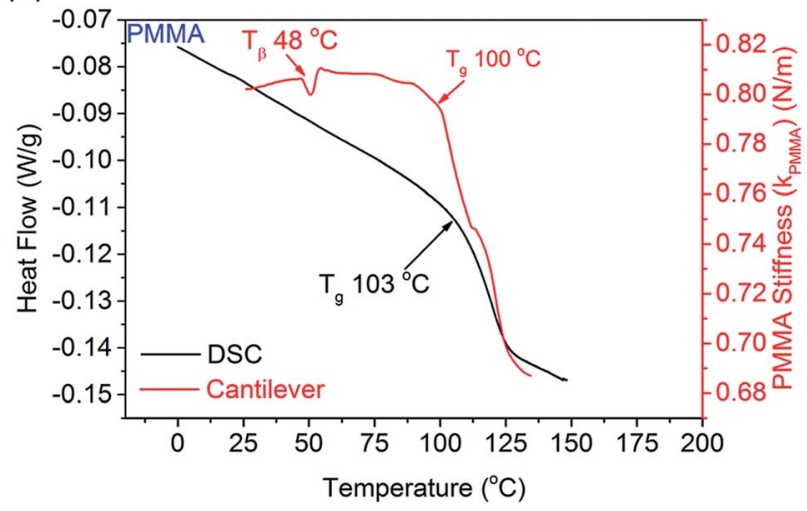

Fig. 4 Heat flow (from DSC) and calculated stiffness of (a) PLA and (b) PMMA are plotted as functions of temperature. 


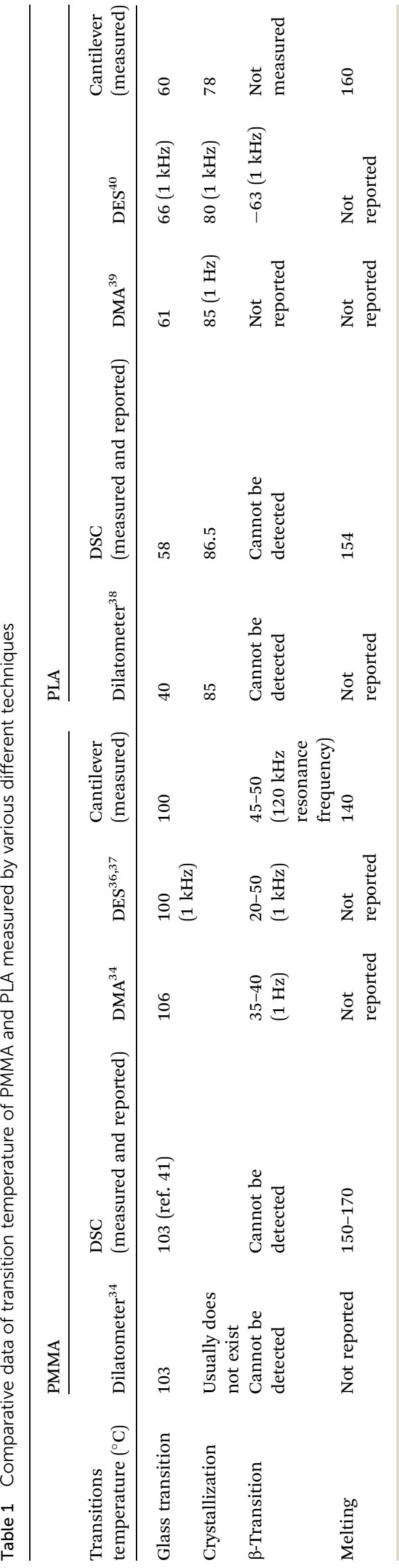

The quality factor of the empty cantilevers was found to be $\sim 6000$. However, after loading the polymers into the microchannel, the resonance frequency of the cantilevers decreased by $14-16 \mathrm{kHz}$ and the quality factor reduced to $\sim 20 \%$ of their original values. These changes ensure that the polymer successfully entered the cantilever. Unlike a cantilever (placed inside a liquid), which experiences a large viscous damping, a microchannel cantilever loaded with a viscous polymer maintains a quality factor well above 1000 .

To accurately perform experiments, stability of the device temperature and its effect on the resonance frequency were first quantified by heating the cantilever in a stepwise manner while simultaneously recording the temperature and frequency response. A $4{ }^{\circ} \mathrm{C}$ step was applied to each cantilever and their corresponding temperature and frequency responses were recorded as shown in Fig. 3a. Temperature stability was achieved after $\sim 60 \mathrm{~s}$. However, as shown in Fig. 3a, the resonance frequency of the cantilever is material dependant and show stabilization times of 100,150 , and $270 \mathrm{~s}$ for the empty $\left(\tau_{c, \text { empty }}\right)$, $\operatorname{PLA}\left(\tau_{\mathrm{c}+\mathrm{PLA}}\right)$, and PMMA $\left(\tau_{\mathrm{c}+\mathrm{PMMA}}\right)$ filled cantilevers, respectively. Therefore, for subsequent measurements we have used 100, 180 and $300 \mathrm{~s}$ as stabilization times for the empty, PLA, and PMMA filled cantilevers respectively. The variation in time constant is directly related to the specific heat capacities of the cantilever material (SiN) and the polymers (PMMA and PLA) stored within. The trend in the observed time constants, $\tau_{\mathrm{c}, \mathrm{empty}}<\tau_{\mathrm{c}+\mathrm{PLA}}<$ $\tau_{\mathrm{c}+\mathrm{PMMA}}$, follows the samples' specific heat capacity as $c_{\mathrm{p}, \mathrm{SiN}}<$ $c_{\mathrm{p}, \text { SiN+PLA }}<c_{\mathrm{p}, \mathrm{SiN}+\mathrm{PMMA}}{ }^{31,32}$

The stabilization time for the resonance frequency of the cantilever (filled with PLA or PMMA) is much longer than $60 \mathrm{~s}$. If a measurement is performed by achieving full stabilization $(3 \tau)$ in the resonance frequency, each measurement would take multiple hours. In order to reduce the measurement time and maintain the detection of small transitions (such as $T_{\beta}$ ), the cantilever was heated at the rate of $4{ }^{\circ} \mathrm{C} \mathrm{min}^{-1}$. This heating rate and stabilization time enabled rapid measurement with sufficient sensitivity and resolution.

Temperature dependant resonance spectra of the empty and polymer-filled cantilevers were measured and plotted as shown in Fig. 3b. In the case of the empty cantilevers, the resonance frequency initially increases with temperature, but no significant changes occur at higher temperatures. This is in stark contrast to the results from the polymer-filled cantilevers, which show a significant reduction in the resonance frequency at specific temperatures, corresponding to various thermal transitions. It is evident that the polymers undergo thermal transitions with increasing temperature, which reduces the effective stiffness of the filled cantilever, resulting in a reduction in the resonance frequency of the cantilever.

Further, the stiffness of the polymer inside the cantilever was determined using eqn (3) and plotted in Fig. 4a and b. Considering that the total mass $\left(m_{\mathrm{p}}+m_{\mathrm{c}}\right)$ and the stiffness $\left(k_{\mathrm{c}}\right)$ of the cantilever material (SiN) are constant, the stiffness of the polymer follows the same trend as that of the resonance frequency of the cantilever. For a comparison, Fig. 4a shows the stiffness of the PLA sample (extracted from the results of the cantilever), as well as the measurements carried out with DSC. 
The overall stiffness of the PLA-filled cantilever initially increases (up to $60{ }^{\circ} \mathrm{C}$ ), which is most likely due to a release of stress (in the silicon nitride). At $\sim 60{ }^{\circ} \mathrm{C}$, the stiffness of the cantilever decreases sharply which is the onset of $T_{\mathrm{g}}$ of PLA. Here the resonance frequency of the cantilever decreases by $60 \mathrm{~Hz}$. The results from DSC indicate that the $T_{\mathrm{g}}$ of the sample lies within the same region. As the temperature is increased further, $k_{\text {PLA }}$ starts to increase at $78{ }^{\circ} \mathrm{C}$, which indicates the onset of crystallization ( $T_{\mathrm{c}}$ ) of PLA. This can be explained as due to an increase in the elastic modulus of PLA during crystallization, which in turn increases the stiffness of the polymer. The $T_{\mathrm{c}}$ of the sample is also evident at $\sim 87^{\circ} \mathrm{C}$ according to the data obtained from DSC. Melting point of the sample is determined by increasing the temperature in a continuous fashion. The onset of melting appears at $\sim 154{ }^{\circ} \mathrm{C}$ (compared to $160{ }^{\circ} \mathrm{C}$ obtained from DSC) as seen by a sharp decrease in $k_{\mathrm{PLA}}$. Upon melting, the viscosity of the sample decreases which explains the reduction in its stiffness.

Fig. 4b shows the thermal transitions of PMMA, measured by the cantilever as well as DSC. As the cantilever is heated, the sample goes through a small but recoverable change in its stiffness $\left(k_{\mathrm{PMMA}}\right)$ at $48{ }^{\circ} \mathrm{C}$. Such a change is not visible in the data obtained using the DSC. This can be attributed to the $\beta$ transition of PMMA and can be explained as due to hindrance of the molecular motion of the $-\mathrm{COOCH}_{3}$ group (attached to the main chain $\mathrm{C}-\mathrm{C}$ bond). ${ }^{33}$ Though this transition cannot be probed using DSC, it can be measured by DMA and DEA, as reported in literature. ${ }^{34,35}$ As the PMMA filled cantilever is further heated to $\sim 100{ }^{\circ} \mathrm{C}$, a sharp reduction in $k_{\mathrm{PMMA}}$ occurs, which is identified as the $T_{\mathrm{g}}$ of PMMA. Such a change is also observed in the DSC data at $103{ }^{\circ} \mathrm{C}$. Any small discrepancies between the temperatures reported between these two techniques may be due to the method used to determine the temperature within the microchannel.

Overall, the thermal transitions of these two polymers measured by suspended microchannel cantilever are in excellent agreement with established techniques such as dilatometry, DMA, and DES as reported in literature as shown in Table 1.

\section{Conclusions}

A suspended microchannel cantilever has been developed to use as a thermal analyzer of picogram amount of sample. Thermal transitions $\left(T_{\beta}, T_{\mathrm{g}}, T_{\mathrm{c}}\right.$ and $\left.T_{\mathrm{m}}\right)$ of picogram amounts of polymer samples were determined by monitoring the resonance response of the microchannel cantilever. The results show an excellent agreement with the data recorded by DSC and other established techniques reported in literature. With further optimization, the microchannel cantilever has the potential to be a precise and convenient tool for thermomechanical characterization of a plethora of different expensive sample types at nano to picoscale.

\section{Acknowledgements}

This work was supported by the Canada Excellence Research Chair (CERC) program. Authors acknowledge Mr Priyesh Dhandaria for the useful discussions.

\section{References}

1 H. Park and K. Park, in Polymers of Biological and Biomedical Significance, American Chemical Society, 1993, ch. 1, vol. 540, pp. 2-15.

2 F. Hussain, M. Hojjati, M. Okamoto and R. E. Gorga, J. Compos. Mater., 2006, 40, 1511-1575.

3 J. Qiao, M. Guo, L. Wang, D. Liu, X. Zhang, L. Yu, W. Song and Y. Liu, Polym. Chem., 2011, 2, 1611-1623.

4 G. Maier, Mater. Today, 2001, 4, 22-33.

5 A. Akelah, in Functionalized Polymeric Materials in Agriculture and the Food Industry, Springer, US, Boston, MA, 2013, pp. 195-248.

6 Z. Hu, M. Tian, B. Nysten and A. M. Jonas, Nat. Mater., 2009, 8, 62-67.

7 A. S. Arico, P. Bruce, B. Scrosati, J.-M. Tarascon and W. van Schalkwijk, Nat. Mater., 2005, 4, 366-377.

8 N. Gadegaard, S. Mosler and N. B. Larsen, Macromol. React. Eng., 2003, 288, 76-83.

9 B. Wunderlich, Thermal Analysis of Polymeric Materials, Springer, New York, 2005.

10 J. Heijboer, Int. J. Polym. Mater., 1977, 6, 11-37.

11 C. Schick, Anal. Bioanal. Chem., 2009, 395, 1589-1611.

12 B. Wunderlich, J. Therm. Anal., 1996, 46, 643-679.

13 D. V. Suriapparao, D. K. Ojha, T. Ray and R. Vinu, J. Therm. Anal. Calorim., 2014, 117, 1441-1451.

14 K. P. Menard, Dynamic Mechanical Analysis: A Practical Introduction, CRC Press, Boca Raton, 1999.

15 A. Y. Olkhov and B. Jurkowski, J. Therm. Anal. Calorim., 2005, 81, 489-500.

16 A. Schönhals, Dielectric Spectroscopy on the Dynamics of Amorphous Polymeric Systems, Novocontrol, 1998.

17 Y. Changyong, Y. Minhyuk, K. Seonghwan, J. Namchul, L. Sang-Hoon, L. Moonchan, R. Shi-Woo, T. Thomas and J. Sangmin, Jpn. J. Appl. Phys., 2012, 51, 08KB07.

18 S. Bose, S. Schmid, T. Larsen, S. S. Keller, P. Sommer-Larsen, A. Boisen and K. Almdal, ACS Macro Lett., 2014, 3, 55-58.

19 J. Fritz, Analyst, 2008, 133, 855-863.

20 J.-P. McGovern, W. Y. Shih, R. Rest, M. Purohit, Y. Pandya and W.-H. Shih, Analyst, 2008, 133, 649-654.

21 N. Jung and S. Jeon, Macromolecules, 2008, 41, 9819-9822.

22 M. Yun, N. Jung, C. Yim and S. Jeon, Polymer, 2011, 52, 41364140.

23 S. Bose, S. S. Keller, T. S. Alstrøm, A. Boisen and K. Almdal, Langmuir, 2013, 29, 6911-6919.

24 J. Kim, J. Song, K. Kim, S. Kim, J. Song, N. Kim, M. F. Khan, L. Zhang, J. E. Sader, K. Park, D. Kim, T. Thundat and J. Lee, Nano Lett., 2016, 16, 1537-1545.

25 M. F. Khan, S. Schmid, P. E. Larsen, Z. J. Davis, W. Yan, E. H. Stenby and A. Boisen, Sens. Actuators, B, 2013, 185, 456-461.

26 M. F. Khan, S. Kim, D. Lee, S. Schmid, A. Boisen and T. Thundat, Lab Chip, 2014, 14, 1302-1307.

27 N. Miriyala, M. F. Khan and T. Thundat, Sens. Actuators, B, 2016, 235, 273-279.

28 Idemat, Matebase, http://www.matbase.com.

29 Sigma-Aldrich, PMMA, http:/www.sigmaaldrich.com/ catalog/product $/$ sial $/ 81489$ ? lang $=$ en\&region $=$ CA. 
30 W. Weaver Jr, S. P. Timoshenko and D. H. Young, Vibration problems in Engineering: Chapter 1, Wiley, 1990.

31 M. Pyda, R. C. Bopp and B. Wunderlich, J. Chem. Thermodyn., 2004, 36, 731-742.

32 D. Liu and C. Zhong, Polym. J., 2002, 34, 954-961.

33 T. Hayashi and K. Fukao, Phys. Rev. E: Stat., Nonlinear, Soft Matter Phys., 2014, 89, 022602.

34 A. Goswami, A. M. Umarji and G. Madras, Polym. Adv. Technol., 2012, 23, 1604-1611.

35 M. S. Ardi, W. Dick and J. Kubát, Colloid Polym. Sci., 1993, 271, 739-747.

36 A. Goswami, A. M. Umarji and G. Madras, Polym.-Plast. Technol. Eng., 2013, 52, 485-494.
37 P. S. Gill, C. L. Marcozzi and I. F. Groves, The characterization of poly(methyl methacrylate), (PMMA), by dielectric analysis and the study of the effects of beta-alkyl substitution on the dielectric properties, Sixth International Conference on Dielectric Materials, Measurements and Applications, 1992.

38 N. Nakayama and T. Hayashi, Polym. Degrad. Stab., 2007, 92, 1255-1264.

39 TA-Instruments, Thermal Analysis Investigation of a Poly(Lactic Acid) Biodegradable Plastic, TA 384.

40 J. Leng, P. J. Purohit, N. Kang, D.-Y. Wang, J. Falkenhagen, F. Emmerling, A. F. Thünemann and A. Schönhals, Eur. Polym. J., 2015, 68, 338-354.

41 A. Goswami, A. M. Umarji and G. Madras, J. Appl. Polym. Sci., 2010, 117, 2444-2453. 